\title{
The magnetic field structure in NGC 253 in presence of a galactic wind
}

\author{
Volker Heesen $^{1}$, M. Krause ${ }^{2}$, R. Beck ${ }^{2}$, and R.-J. Dettmar ${ }^{1}$ \\ ${ }^{1}$ Astronomisches Institut der Ruhr-Universität Bochum, 44780 Bochum, Germany \\ email: heesen@astro.rub.de,dettmar@astro.rub.de \\ ${ }^{2}$ Max-Planck-Institut für Radioastronomie, 53121 Bonn, Germany \\ email: rbeck@mpifr-bonn.mpg.de, mkrause@mpifr-bonn.mpg.de
}

\begin{abstract}
We present radio continuum polarimetry observations of the nearby edge-on galaxy NGC 253 which possesses a very bright radio halo. Using the vertical synchrotron emission profiles and the lifetimes of cosmic-ray electrons, we determined the cosmic-ray bulk speed as $300 \pm 30 \mathrm{~km} \mathrm{~s}^{-1}$, indicating the presence of a galactic wind in this galaxy. The large-scale magnetic field was decomposed into a toroidal axisymmetric component in the disk and a poloidal component in the halo. The poloidal component shows a prominent X-shaped magnetic field structure centered on the nucleus, similar to the magnetic field observed in other edge-on galaxies. Faraday rotation measures indicate that the poloidal field has an odd parity (antisymmetric). NGC 253 offers the possibility to compare the magnetic field structure with models of galactic dynamos and/or galactic wind flows.
\end{abstract}

Keywords. Galaxies: halos - galaxies: individual (NGC253) - galaxies: magnetic fields - galaxies: spiral - radio continuum: galaxies

\section{Introduction}

Gaseous halos are a common property of star forming galaxies. The abundance of hot coronal gas in the halo requires heating from the star forming disk and a transport of energy from the disk into the halo. This energy transport has been discussed as galactic fountains or chimneys (Field et al. 1969, Norman \& Ikeuchi 1989). The relativistic cosmicray gas has an adiabatic index of $4 / 3$ compared to $5 / 3$ of the (single atomic) hot gas; this results in a larger pressure scaleheight of the cosmic-ray gas. The magnetic field has an even larger pressure scaleheight (Beck 2009). The pressure contributions of the cosmic rays and the magnetic field are thus dominating in the halo and can accelerate the outflow. This led to the model of a cosmic-ray driven wind that causes acceleration of the flow accelerates further away from the disk (Breitschwerdt et al. 1991).

The existence of radio halos around many nearby galaxies shows the presence of cosmic rays and magnetic fields. Cosmic rays spiral around magnetic field lines and follow them from the disk into the halo. Hence, a vertical component of the large-scale magnetic field can enhance the vertical cosmic-ray transport. How to generate vertical fields is still an open question. Parker (1992) suggested that field lines start to overlap and reconnect to form "open" field lines that connect the disk with the halo. Another possibility is the mean field $\alpha \Omega$-dynamo, which creates dipolar or quadrupolar field configurations. Furthermore, the galactic wind may shape the magnetic field and align with it. This may particularly hold for superwinds, which originate in a region of very active star formation (starburst).

As shown by X-ray observations, NGC 253 has a superwind and is an ideal object to study the interaction between the superwind and the magnetic field. There is a nuclear 
plume of $\mathrm{H} \alpha$ and X-ray emitting gas (Strickland et al. 2000, Bauer et al. 2007) where the outflow velocity was directly measured by spectroscopy as $390 \mathrm{~km} \mathrm{~s}^{-1}$ (Schulz \& Wegner 1992). The connection between the heated gas in the halo, which extends far from the disk, and the the superwind is likely as suggested by numerical simulations of a centrally driven wind (Suchkov et al. 1994). A detailed study of this galaxy is possible because it is nearby $\left(D=3.94 \mathrm{Mpc}\right.$, Karachentsev et al. $\left.2003,30^{\prime \prime}=600 \mathrm{pc}\right)$. With an inclination angle of $78^{\circ}$ the galaxy's disk is only mildly edge-on. But it contains a very bright nucleus which complicates the data reduction of radio continuum observations.

\section{Observations}

Our new results are based on a deep mosaic with the VLA at $\lambda 6.2 \mathrm{~cm}$. This wavelength is optimal to study magnetic fields in galactic halos, because the polarization is not too much affected by Faraday rotation and depolarization. On the other hand, the contribution from the thermal emission is still small $(<10 \%)$ and the vertical extent is large. The largest angular scale that can be well imaged with the VLA at $\lambda 6.2 \mathrm{~cm}$ is $5^{\prime}$. We used a $\lambda 6.3 \mathrm{~cm}$ map observed with the $100-\mathrm{m}$ Effelsberg telescope to fill in the missing zero-spacing flux.

Additionally we used VLA maps at $\lambda 20 \mathrm{~cm}$ (Carilli et al. 1992) and at $\lambda 90 \mathrm{~cm}$ (Carilli 1996). In order to correct the magnetic field for Faraday rotation we observed the galaxy with the 100-m Effelsberg telescope at $\lambda 3.6 \mathrm{~cm}$. A detailed description of the observations and the data reduction can be found in Heesen (2008).

\section{Morphology}

In Fig. 1 we present the $\lambda 6.2 \mathrm{~cm}$ total power radio continuum emission from the combined VLA and Effelsberg observations. The vectors show the Faraday corrected orientation of the large-scale magnetic field. The emission shows a prominent dumbbell shaped radio halo. The vertical extent is smallest near the center and increases further out in the disk. A detailed analysis of the vertical emission profiles shows that the scaleheights are smallest near the center.

The distribution of the polarized emission shown in Fig. 2 is more concentrated to the inner disk than the total power emission. It is extending into the halo particularly in the inner disk. The orientation of the magnetic field is disk-parallel in the inner part of the disk but turns away from the disk in the outer part. The vertical component even dominates at the "radio spur" (R.A. $=00^{\mathrm{h}} 47^{\mathrm{m}} 50^{\mathrm{s}}$, Dec. $=-25^{\circ} 17^{\mathrm{m}} 00^{\mathrm{s}}$ ), where the magnetic field orientation is almost perpendicular to the disk.

\section{Cosmic-ray bulk speed}

The dumbbell shaped radio halo shows that the vertical extension is smallest in the inner part of the disk where the magnetic field strength is highest. Synchrotron losses are the dominant energy loss of the cosmic-ray electrons (Heesen et al. 2009a). The synchrotron lifetime

$$
t_{\mathrm{syn}}=\frac{8.352 \times 10^{9} \mathrm{yr}}{(\nu / 16.1 \mathrm{MHz})^{1 / 2}\left(\mathrm{~B}_{\perp} / \mu \mathrm{G}\right)^{3 / 2}}
$$

depends on the total magnetic field strength $B_{\perp}$ perpendicular to the line-of-sight and on the observation frequency $\nu$. Thus, it is smallest in the central part of the disk. To 


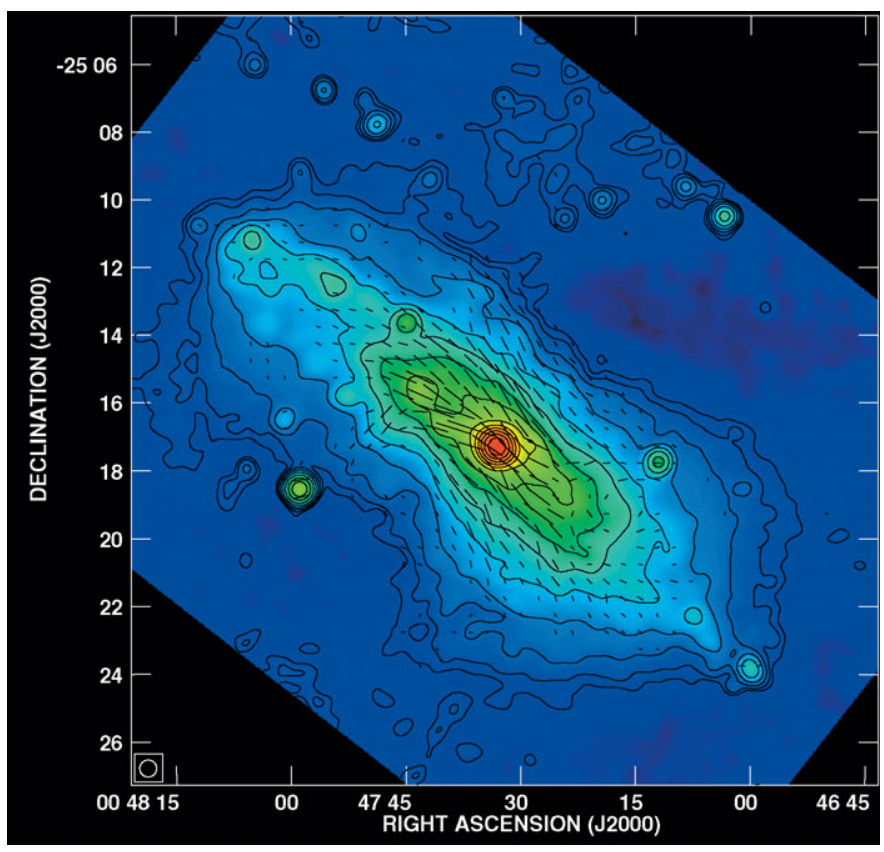

Figure 1. Total power radio continuum at $\lambda 6.2 \mathrm{~cm}$ obtained from the combined VLA + Effelsberg observations with $30^{\prime \prime}$ resolution. Contours are at 3 , $6,12,24,48,96,192,384$, $768,1536,3077,6144,12288$, and $24576 \times$ the rms noise of $30 \mu \mathrm{Jy} /$ beam. The overlaid vectors indicate the orientation of the Faraday corrected regular magnetic field. A vector length of $1^{\prime \prime}$ is equivalent to $12.5 \mu \mathrm{Jy} /$ beam polarized intensity.

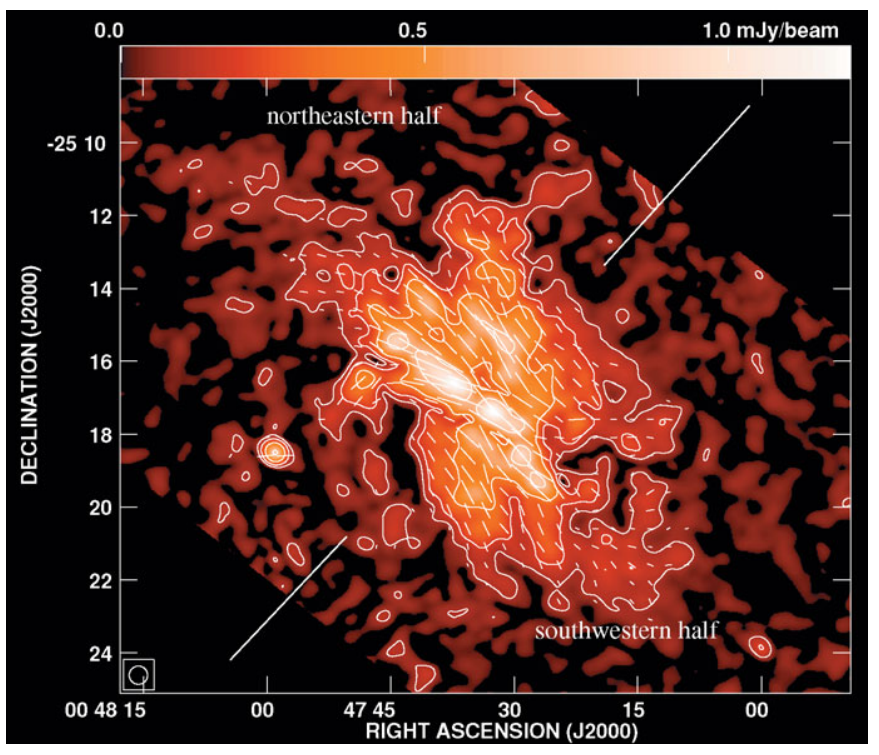

Figure 2. Polarized intensity obtained from the combined VLA + Effelsberg observations at $\lambda 6.2 \mathrm{~cm}$ with $30^{\prime \prime}$ resolution. Contours are at $3,6,12$, and $24 \times$ the rms noise level of $30 \mu \mathrm{Jy} /$ beam. The overlaid vectors indicate the orientation of the Faraday corrected regular magnetic field. A vector length of $1^{\prime \prime}$ is equivalent to $12.5 \mu \mathrm{Jy} /$ beam polarized intensity.

calculate the electron lifetime we have to add the timescale $t_{\mathrm{a}}$ of the adiabatic losses:

$$
\frac{1}{t_{\mathrm{e}}}=\frac{1}{t_{\mathrm{a}}}+\frac{1}{t_{\mathrm{syn}}} \text {. }
$$

Figure 3 shows the scaleheight of the synchrotron emission as a function of the electron lifetime. The linear dependence, particularly in the northeastern halo of the galaxy (c.f. Fig. 2), suggests a definition of an average cosmic-ray bulk speed

$$
\mathrm{v}=\frac{h_{\mathrm{e}}}{t_{\mathrm{e}}}
$$



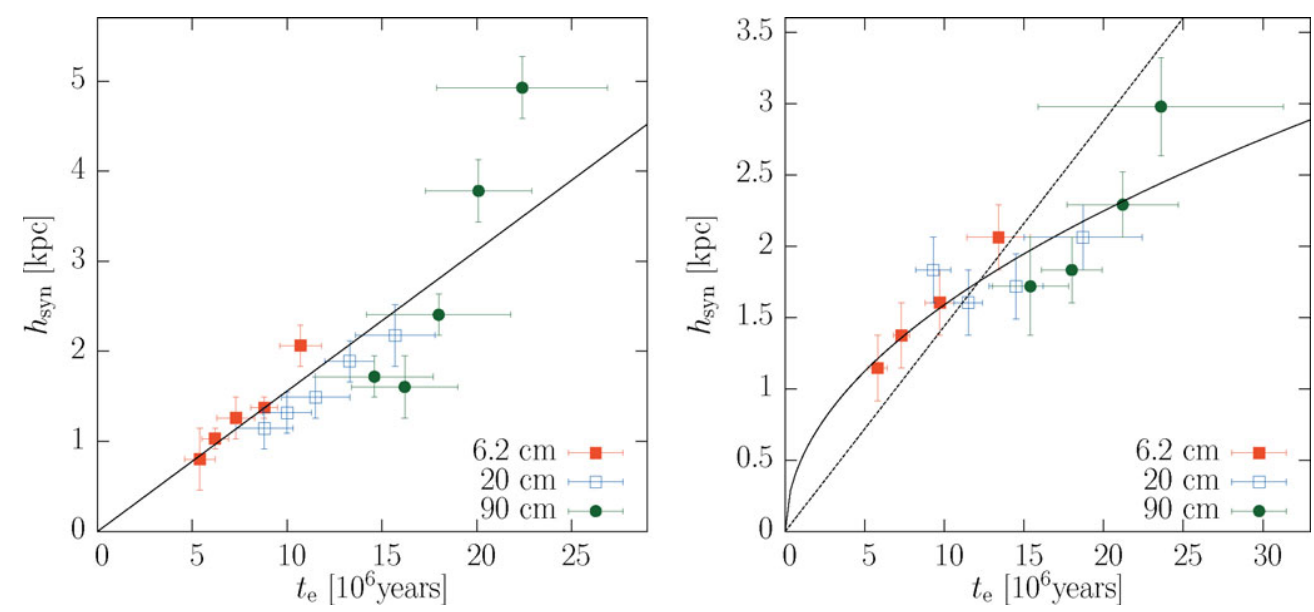

Figure 3. Scaleheight $h_{\mathrm{syn}}$ of the thick radio disk as a function of the electron lifetime $t_{\mathrm{e}}$ in the northeastern halo (left) and in the southwestern halo (right). The linear fit is the theoretical expectation for a convective cosmic-ray transport with a constant bulk speed. The fit $h_{\mathrm{e}} \propto \sqrt{t_{\mathrm{e}}}$ is the theoretical expectation for a diffusive cosmic-ray transport.

where $h_{\mathrm{e}}$ is the electron scaleheight (twice the synchrotron scaleheight). In the northeastern halo, we find a cosmic-ray bulk speed of $300 \pm 30 \mathrm{~km} \mathrm{~s}^{-1}$. Because the cosmic-ray bulk speed does not depend on the electron energy, the cosmic-ray transport is convective. In the southwestern halo, the scaleheight can be better described by $h_{\mathrm{e}} \propto \sqrt{t_{\mathrm{e}}}$. The cosmic-ray bulk speed increases with the electron energy, which indicates a larger role of diffusion in this halo part. In case of pure diffusion we have a diffusion coefficient of $\kappa=2.0 \pm 0.2 \times 10^{29} \mathrm{~cm}^{2} \mathrm{~s}^{-1}$.

Because the cosmic-ray electrons lose their energy via synchrotron radiation, their energy spectral index increases during their lifetime. This is visible as a steepening of the radio spectral index in the halo. We detected an almost linear dependence of the radio spectral index on the distance from the disk. From this we derived $170 \mathrm{~km} \mathrm{~s}^{-1}$ as a lower limit for the average cosmic-ray bulk speed.

\section{Structure of the magnetic field}

Our model of an axisymmetric spiral, disk-parallel toroidal magnetic field (Fig.4) represents the observations well. We chose the inclination angle of the optical disk and a best-fit spiral pitch angle of $25^{\circ}$, which is similar to the pitch angle of $20^{\circ}$ of the optical spiral arms. At the locations where the toroidal magnetic field is weak we also observe a vertical poloidal field (Fig. 2). We subtracted the toroidal field model from the observations and obtained the poloidal magnetic field shown in Fig. 5. It has a prominent $\mathrm{X}$-shaped structure centered on the nucleus.

The structure of the poloidal magnetic field seems to be connected to the distribution of the heated gas in the halo. This gas forms a horn-like structure with lobes that are thought to be the walls of a superbubble filled with dilute hot gas. This can be seen in Fig. 5 where the sensitive XMM observations show X-ray emitting gas also inside the bubble. The similarity of the structure of the poloidal magnetic field to that of the superbubble and their alignment can be explained by an interaction between the superwind, driven by the nuclear starburst, and the surrounding medium transported by the disk wind, possibly enhanced by shock waves of the expanding superbubble. 


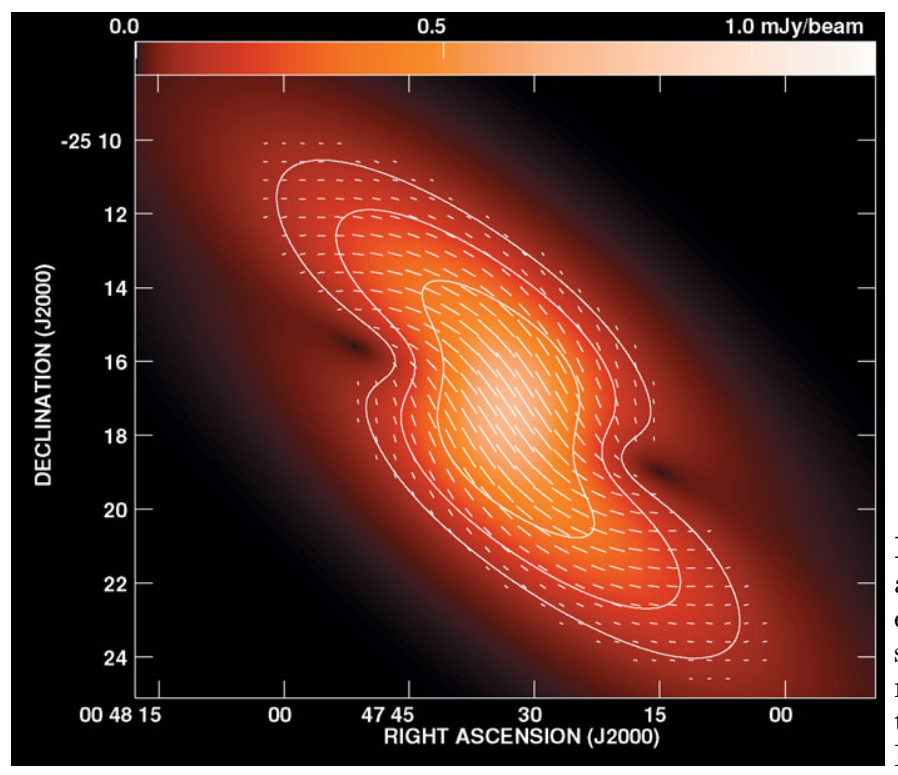

Figure 4. Polarized emission and magnetic field orientation expected for an axisymmetric spiral model for the toroidal magnetic field at $180^{\prime \prime}$ resolution. Contours and vectors as in Fig. 2.

The difference between the polarization angles at $\lambda \lambda 6.2 \mathrm{~cm}$ and $3.6 \mathrm{~cm}$ is due to Faraday rotation. This provides information about the line-of-sight component of the large-scale magnetic field. Krause et al. (1989) showed that the azimuthal variation of rotation measure $(\mathrm{RM})$ can be used to determine the contributions from individual dynamo modes. In NGC 253, the RM is the superposition of the toroidal magnetic field in the disk and the poloidal magnetic field in the halo. Subtracting the model for the disk magnetic field leaves the RM distribution of the poloidal magnetic field. It has the maximum amplitude along the minor axis. We propose a conical configuration for the poloidal magnetic field with an opening angle of $66^{\circ}$ of odd symmetry: the field direction points towards the disk in the southern hemisphere (c.f. 5) and away from the disk in the northern hemisphere (Heesen et al. 2009). The field lines are aligned with the lobes of hot gas in the halo.

\section{Conclusions}

NGC 253 possesses a bright radio halo. The scaleheight of the synchrotron emission depends on the lifetime of the cosmic-ray electrons. This requires a vertical cosmicray transport from the disk into the halo. The disk wind has an average velocity of $300 \pm 30 \mathrm{~km} \mathrm{~s}^{-1}$ and is surprisingly constant over the extent of the disk. The disk wind transports material from the disk into the halo. If this material is the origin of the luminous, heated gas in the halo, it can explain the asymmetry between the northeastern and southwestern halo, as the cosmic-ray transport is much more efficient in the convective northeastern halo than in the diffusive southwestern halo.

In the disk the magnetic field is parallel to the disk. A model of an axisymmetric spiral disk field can explain the observed asymmetries in polarization by a geometrical effect. After subtracting the disk field model from the observations we obtain the poloidal magnetic field in the halo. This field has a prominent X-shaped structure that is also seen in several other edge-on galaxies. For the first time the parity of the large-scale field in any galaxy could be determined, which is odd (antisymmetric) in NGC 253. The X-shape may be explained by interaction of the halo gas transported by the disk wind with the 


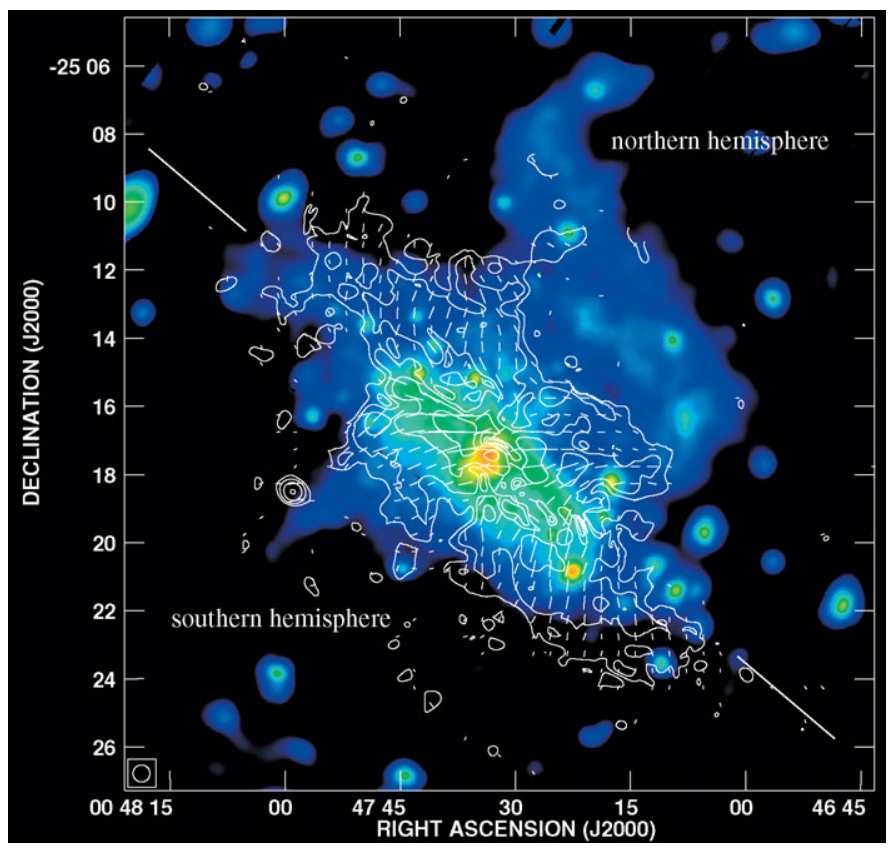

Figure 5. Polarized emission and magnetic field orientation of the poloidal magnetic field overlaid onto diffuse X-ray emission. Contours and vectors as in Fig. 2. The X-ray map is from XMM observations in the energy band 0.5-1.0 keV (Bauer et al. 2008).

superwind from the starburst center. However, several galaxies without a superwind also show X-shaped halo fields (Krause 2008), which needs to be further investigated.

\section{References}

Bauer, M., Pietsch, W., Trinchieri, G., Breitschwerdt, D., Ehle, M., \& Read, A. 2007, A\&A 467, 979

Bauer, M., Pietsch, W., Trinchieri, G., Breitschwerdt, D., Ehle, M., Freyberg, M. J., \& Read, A. M. $2008, A \& A 489,1029$

Beck, R. 2009, these proceedings

Breitschwerdt, D., McKenzie, J. F., \& Voelk, H. J. 1991, A $\& A$ 245, 79

Carilli, C. L., Holdaway, M. A., Ho, P. T. P., \& de Pree, C.G. 1992, ApJ 399, L59

Carilli, C. L. 1996, A\&A 305, 402

Field, G. B., Goldsmith, D. W., \& Habing, H. J. 1969, ApJ 155, L149

Heesen, V. 2008, PhD thesis, Ruhr-Universität Bochum, Germany

Heesen, V., Beck, R., Krause, M., \& Dettmar R.-J., 2009a, A\&AA, in press; arXiv:0812.0346

Heesen, V., Krause, M., Beck, R., \& Dettmar R.-J. 2009b, to be submitted

Karachentsev, I. D., Grebel, E. K., Sharina, M. E., Dolphin, A. E., Geisler, D., Guhathakurta, P., Hodge, P. W., Karachentseva, V. E., Sarajedini, A., \& Seitzer, P. A\&̈A 404, 93

Krause, M., Hummel, E., \& Beck, R. 1989, A\&A 217, 4

Krause, M. 2008, Magnetic Fields in the Universe II, eds. A. Esquivel et al., arXiv:0806.2060

Norman, C. A. \& Ikeuchi, S. 1989, ApJ 345, 372

Parker, E. N. 1992, ApJ 401, 137

Schulz, H. \& Wegner, G. 1992, A\&A 266, 167

Strickland, D. K., Heckman, T. M., Weaver, K. A., \& Dahlem, M. 2000, AJ 120, 2965

Suchkov, A. A., Balsara, D. S., Heckman, T. M., \& Leitherner, C. 1994, ApJ 430, 511 